\title{
UNIVERSAL TRANSITION IN A DYNAMICAL SYSTEM FORCED AT TWO INCOMMENSURATE FREQUENCIES
}

\author{
James P. SETHNA \\ Laboratory of Atomic and Solid State Physics, Cornell University, Ithaca, NY 14583, USA \\ and \\ Institute for Theoretical Physics ${ }^{\dagger}$, University of California, Santa Barbara, CA 93106, USA \\ and \\ Eric D. SIGGIA \\ Laboratory of Atomic and Solid State Physics, Cornell University, Ithaca, NY 14853, USA
}

Received 16 May 1983

Revised manuscript received 26 October 1983

\begin{abstract}
The various routes to chaos are explored for a nonlinear mechanical system mode locked to one of two incommensurate external frequencies. Two types of transitions are seen in our model system. A saddle-node transition with its associated intermittency can occur when the mode locking is lost while the attracting two-torus remains smooth. A less trivial transition can occur in which the attracting torus roughens and the power spectrum of the time series develops singular low frequency components; after the breakdown mode locking persists with noisy small-scale motions about the former torus. At the multicritical point where these two transition lines meet scaling and universal low-frequency power spectra are observed. A renormalization group treatment is proposed. The analysis might also be applicable to transitions in rotationally invariant systems.
\end{abstract}

\section{Introduction}

Certain transitions (bifurcations) in the dynamics of classical mechanical systems are closely related to phase transitions in statistical mechanics. In particular, renormalization group methods developed to study second order phase transitions have been successfully applied to several continuous bifurcations [1-6]. The renormalization group in these problems amounts to a decimation of the time series; because the time series is onedimensional, the analysis essentially can be done exactly. The universal predictions about these bi-

${ }^{+}$Address as of August 1, 1983. furcations can be more detailed than those about phase transitions. Not only are there exponents governing the approach to the transition but often $[1-5]$ there is a highly structured singular universal low frequency spectrum at the transition.

In this paper we begin the study of another class of bifurcations. Consider a dissipative, nonlinear physical system forced at two incommensurate frequencies. Forced only at one frequency $\omega_{0}$ the system will often respond at a frequency $p \omega_{0} / q$ commensurate with the forcing. This mode locked state will generically be stable to small variations in the dynamics. In particular, we shall see that it will persist under the introduction of the second frequency $\omega_{2}$.

0167-2789/84/\$03.00 (C) Elsevier Science Publishers B.V.

(North-Holland Physics Publishing Division) 
Let our physical system be represented by a single variable $\phi_{1}$ and our external forces by variables $\phi_{0}$ and $\phi_{2}, 0 \leqslant \phi_{i} \leqslant 2 \pi$. If we measure the state of the system at periodic intervals $2 \pi / \omega_{0}$, we get a Poincaré once return map $f\left(\phi_{1}, \phi_{2}\right)$. We believe the following form is sufficiently general to encompass the (universal) features of the bifurcations we intend to study:

$$
\begin{aligned}
& f\left(\phi_{1}, \phi_{2}\right)=\left(\begin{array}{l}
f_{1}\left(\phi_{1}, \phi_{2}\right) \\
f_{2}\left(\phi_{1}, \phi_{2}\right)
\end{array}\right) \\
& =\left(\begin{array}{c}
\phi_{1}+\omega+a \cos \left(\phi_{1}\right)+b \cos \left(\phi_{1}-\phi_{2}\right) \\
\phi_{2}+2 \pi \sigma
\end{array}\right) .
\end{aligned}
$$

We define winding numbers $\rho_{1}$ and $\rho_{2}$ for $\boldsymbol{f}$, giving the mean rotation in the $\phi_{1}$ and $\phi_{2}$ directions,

$\rho_{i}=\lim _{n \rightarrow \infty}\left[\left(f^{n}\right)_{i}\left(\phi_{1}, \phi_{2}\right)-\phi_{i}\right] /(2 \pi n)$.

In eq. (1.1), $\rho_{2} \equiv \sigma$. We confine our analysis to the golden mean $\sigma=(\sqrt{ } 5-1) / 2$. Extensions to other good irrationals should follow as in ref. 2; why the golden mean should be experimentally optimum is also discussed there.

At $b=0$ the system is forced at only one frequency; for $|a|<|\omega|$ the system is mode locked to that frequency. That is, the map $f_{1}$ has a stable fixed point at $\phi_{1}=s=\arccos (-\omega / a)$ and unstable fixed point at $\phi_{1}=u=2 \pi-s$; the winding number $\rho_{1}=0$. For small $b$ these turn into curves $\phi_{1}=s\left(\phi_{2}\right)$ and $\phi_{1}=u\left(\phi_{2}\right)$ (fig. 1) which in the original state space form an attracting and a repelling torus. This article is devoted to a thorough study of the destruction of the stable curve $s\left(\phi_{2}\right)$ as we change the parameters $a, b$, and $\omega$.

The system described in eq. (1.1) should be generic (as described above) in the class of nonlinear dissipative dynamical systems forced at two frequencies. (More precisely, eq. (1.1) describes generic nonlinear dissipative systems with two neutral directions representing periodic forces and all but one of the remaining directions strongly contracting. As in Feigenbaum period doubling, we believe this noninvertible map has the universal

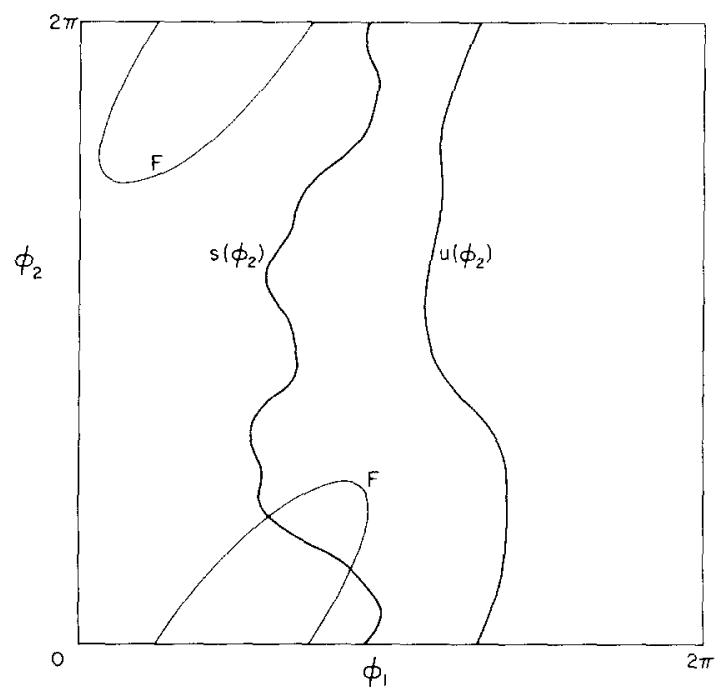

Fig. 1. Stable and unstable curves. The stable and unstable curves found by iterating $f$ of eq. (1.1) with $a=0.5, b=0.9$, $\omega=0.4$ and as always $\sigma=(\sqrt{ } 5-1) / 2$. The curves $s$ and $u$ are the Poincare sections of the stable and unstable torus in the original (continuous time) dynamical system. Along the football-shaped curve $F$ the Jacobian $\partial f_{1} / \partial \phi_{1}$ of $f$ is zero, and $f$ does not have an inverse. Under iterations, the flow is a motion away from $u$ and into the curve $s$, superimposed upon a uniform rotation in the $\phi_{2}$ direction. As one approaches the saddle-node transition from this state, the curve $s$ will first leavs the region bounded by $F$ and then the curves $s$ and $u$ wil smoothly and uniformly coalesce.

features of dissipative invertible systems in highe: dimensions.) For arbitrary flows on a three-toru: it is of course not generic, since $f_{2}$ should includi an additive periodic function of $\phi_{1}$ and $\phi_{2}$. $A$ mode-locked state on a three-torus with two in commensurate frequencies whose ratio is kept fixer can break down in a variety of ways. For som parameters the embedded two-torus shouk roughen following the same universal route as th dissipative annular maps of refs. 2 and 3. Alterna tively, the two-torus can "collide" with its unstabl counterpart in a higher dimensional analogue o the saddle-node bifurcation [5] (see also section 2.2). It is also possible that a thorough renor malization group analysis would show the generi nonlinear terms in $f_{2}$ can be irrelevant, leading on back to the system described in eq. (1.1). There ar several qualitatively different transitions tha 
emerge from (1.1) that we analyze below. Whether at any of these the nonlinear terms in $f_{2}$ are irrelevant remains to be seen.

In general (and we believe in eq. (1.1)) flows on a three-torus are mode locked on an open dense subset of parameter space. When the parameters are adjusted so that the rotation rates of the three phases are suitably incommensurate, then Ruelle, Takens and Newhouse [7] have shown that arbitrarily close to such a flow in the space of all possible flows there is an open set on which the motion is described by a truely chaotic attractor. (The "chaos" in their construction is confined to very low frequencies.) On the other hand, one could hope to use a KAM-like approach to show by varying two parameters consistently as the stress (nonlinearity) on the system is increased that a quasiperiodic flow on a three-torus can be kept quasiperiodic-i.e., non-chaotic [8]. (Arnold has shown this for a two-torus [9].) We numerically checked that eq. (1.1), with $a=0.5, b=0.2$ and $\sigma=\rho_{2}=(\sqrt{ } 5-1) / 2$ can be tuned by varying $\omega$ to achieve $\rho_{1}=\sqrt{ } 2-1$; the spectrum numerically consists of sharp peaks (quasiperiodic) with no observed chaos. Thus in this (non-generic) map, quasiperiodic flow on a three torus can be maintained by varying one parameter.

Rand [10] has shown that in systems with circular symmetry flow on a two-torus will not mode lock. This makes (1.1) an attractive model of Couette flow in the modulated wavy vortex regime, though it is certainly not generic for rotationally symmetric systems (e.g., we have assumed the attractor lies on a three torus in the chaotic regime). Also, one can put this system in a parameter regime where there is naturally periodic motion and apply one external incommensurate force.

Another setting in which systems of the form (1.1) naturally arise is in the spectra of a onedimensional Schrödinger equation with a quasiperiodic potential. Consider the equation

$\left[-\frac{\partial^{2}}{\partial x^{2}}+U\left(\omega_{0} x, \omega_{2} x\right)\right] \psi=E \psi$,

where $U$ is periodic with period $2 \pi$ in both its arguments. It has been shown [11] that there are extended eigenstates of (1.3), with the form

$\psi(x)=\mathrm{e}^{\mathrm{i} \omega_{1} x} \chi\left(\omega_{0} x, \omega_{2} x\right)$

with $\chi$ again $2 \pi$ periodic in its arguments. If one thinks of the Schrödinger eq. (1.3) as an autonomous system of differential equations in "time",

$\frac{\partial \phi_{0}}{\partial t}=\omega_{0}, \quad \frac{\partial \phi_{2}}{\partial t}=\omega_{2}, \quad \frac{\partial^{2} \psi}{\partial t^{2}}=\left[E-U\left(\phi_{1}, \phi_{2}\right)\right] \psi$,

then the proof of (1.4) is equivalent to the existence of quasiperiodic motion on a three-torus $\left(\phi_{0}, \phi_{2}\right.$ and $\phi_{1}=\arg (\psi)$ with $|\psi|$ a smooth function of $\phi_{0}$, $\phi_{1}$ and $\phi_{2}$ ). The energy $E$ is a parameter analogous to $\omega$ in eq. (1.1); as it is varied, $\phi_{1}$ mode locks to $\phi_{0}$ and $\phi_{2}$ on an open dense set (gaps in the spectrum), and is incommensurate on the complement (bands). Of course since the $\psi$ equation is linear, the evolution of $\phi_{1}$ implicit in (1.5) is non-generic. Nonetheless, this system may share some fixed points with eq. (1.1).

\section{Qualitative properties}

\subsection{The mode-locked region}

As we vary the parameters $a, b$ and $\omega$, the stable curve $\phi_{1}=s\left(\phi_{2}\right)$ of eq. (1.1) deforms (see fig. 1), until it is eventually destroyed. Fig. 2 is a phase diagram for $a=1 / 2$ showing the mode-locked region where $s\left(\phi_{2}\right)$ is smooth and has zero winding number in the $\phi_{1}$ direction $\left(\rho_{1}=0\right)$. There are two ways in which $s$ is destroyed. Along the side boundaries we shall show that $s$ disappears in a saddle-node bifurcation. That is, $s\left(\phi_{2}\right)$ while remaining smooth collides with the unstable curve $u\left(\phi_{2}\right)$ and annihilates in a manner quantitatively equivalent to the familiar coalescence of stable and unstable periodic orbits studied in refs. 6. Along the top boundary $s$ becomes crinkled (singular on short lengthscales $=$ long timescales), leading to a 


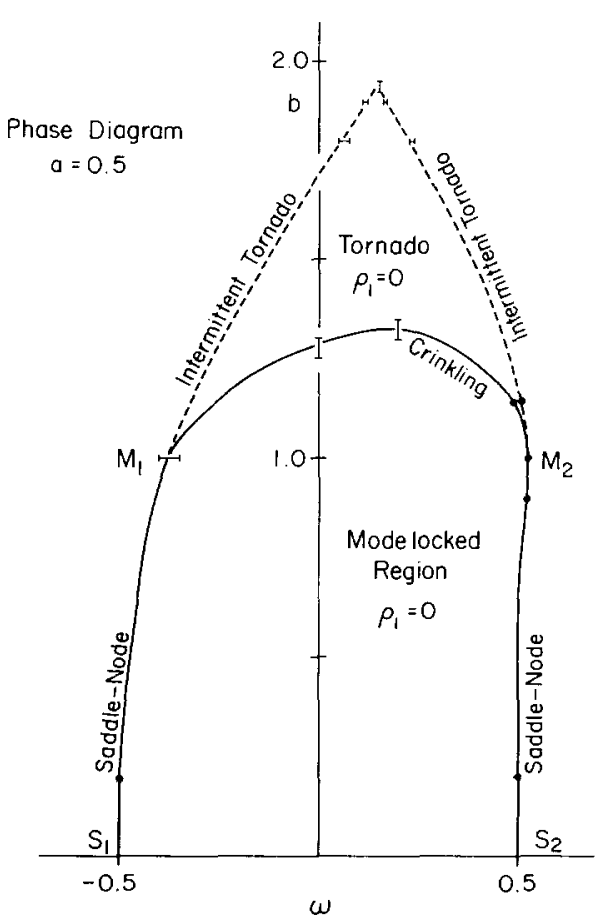

Fig. 2. Phase diagram $a=0.5$. Approximate boundaries for regions of zero winding number in the $\phi_{1}$ direction, for the map $f$ of eq. (1.1) with $a=1 / 2$ and $\sigma=(\sqrt{5}-1) / 2$. The various phases and transitions in this diagram are the subject of this paper. In the mode-locked region the attractor is apparently an analytic curve $\phi_{1}=s\left(\phi_{2}\right)$; in the tornado region the attractor is bounded in the $\phi_{1}$ direction (zero winding number $\rho_{1}$ ) but appears to fill a two-dimensional area. The crinkling and saddle-node transitions are the two-ways in which the mode locked state $s\left(\phi_{2}\right)$ can break down. The dashed boundary of the tornado region represents the onset of intermittent bursts (winding in the $\phi_{1}$ direction) in the already chaotic tornado state.

chaotic state with zero winding number $\left(\rho_{1}=0\right)$ and a fuzzy, "tornado" like attractor.

First, one might ask why the curve $s$ remains smooth as parameters are varied. Suppose for one value of the parameter $\boldsymbol{p}=(a, b, \omega)$ the map $f^{p}\left(\phi_{1}, \phi_{2}\right)$ of eq. (1.1) maps the smooth curve $s$ into itself. Under a small change $\delta \boldsymbol{p}$ in $\boldsymbol{p}$, we want a new curve $(s+\delta s)\left(\phi_{2}\right)$ close to $s$ which is fixed under $f^{p+\delta p}$. If we define

$$
d\left(\phi_{2}\right)=f_{1}^{p+\delta p}\left(s\left(\phi_{2}-2 \pi \sigma\right), \phi_{2}-2 \pi \sigma\right)-s\left(\phi_{2}\right),
$$

$$
\begin{aligned}
\mathrm{D} f\left(\phi_{2}\right) & =\frac{\partial f_{1}}{\partial \phi_{1}}\left(s\left(\phi_{2}\right), \phi_{2}\right) \\
& =1-a \sin \left(s\left(\phi_{2}\right)\right)-b \sin \left(s\left(\phi_{2}\right)-\phi_{2}\right),
\end{aligned}
$$

then $d$ and $\mathrm{D} f$ are also smooth functions of $\phi_{2}$. To first order in $\delta p, \delta s$ must satisfy

$$
\begin{aligned}
\delta s\left(\phi_{2}\right)= & d\left(\phi_{2}\right)+\mathrm{D} f\left(\phi_{2}-2 \pi \sigma\right) \delta s\left(\phi_{2}-2 \pi \sigma\right) \\
= & \sum_{m=0}^{n-1} d\left(\phi_{2}-2 \pi m \sigma\right) \prod_{k=1}^{m} \mathrm{D} f\left(\phi_{2}-2 \pi k \sigma\right) \\
& +\delta s\left(\phi_{2}-2 \pi n \sigma\right) \prod_{k=1}^{n} \mathrm{D} f\left(\phi_{2}-2 \pi k \sigma\right) .
\end{aligned}
$$

If $\mathrm{D} f$ has only power-law zeros, we can define the Liapunov exponent (the contraction rate)

$$
\begin{aligned}
\Lambda \equiv & \int_{0}^{2 \pi} \frac{\mathrm{d} \phi_{2}}{2 \pi} \log \mid 1-a \sin \left(s\left(\phi_{2}\right)\right) \\
& -b \sin \left(s\left(\phi_{2}\right)-\phi_{2}\right) \mid
\end{aligned}
$$

If $\Lambda<0$, then for good irrationals $\sigma$ the infinite product $\Pi_{K=1}^{N} \mathrm{D} f\left(\phi_{2}-k \sigma\right)$ should converge to zero like $\mathrm{e}^{N A}$, and $\delta s$ should be a smooth function of $\phi_{2}$ (since $\delta s$ in eq. (2.3) is essentially a finite (geometrically converging) sum of smooth functions). Numerically, $\Lambda \rightarrow 0$ on the boundaries of the mode-locked region in fig. 2 . We conjecture that for good irrationals $\sigma, \Lambda=0$ is a necessary condition for the breakdown of mode locking.

We note here that the map $f$ in eq. (1.1) is not invertible when $|a|+|b|>1$. The curve $F$ where the Jacobian $1-a \sin \phi_{1}-b \sin \left(\phi_{1}-\phi_{2}\right)$ of $f$ is zero is a football-shaped region depicted e.g., in fig. 1. $F$ will be an important curve in our analysis.

\subsection{The saddle-node transition}

Across the side boundaries of the mode locked region in fig. 2 , the iterates of the map wind in the 
$\phi_{1}$ direction $\left(\rho_{1} \neq 0\right)$. Between the mode-locked regions, the map can exhibit quasiperiodic motion of three incommensurate frequencies as mentioned in the introduction. Truely chaotic motion (with positive entropy) is only possible when the map is noninvertible. (The Sinai entropy of a map is less than or equal to the sum of its positive characteristic exponents. If a map is invertible, its entropy is equal to that of its inverse. Since one of our characteristic exponents is always zero [in the trivial $\phi_{2}$ direction], if $f$ is invertible its entropy is zero). The map can also lock into modes where 1 , $\rho_{1}$, and $\rho_{2}$ are rationally related; fig. 3 shows a case where $11 \rho_{1}=-1+2 \rho_{2}$. These mode locked states have phase diagrams qualitatively similar to fig. 2 , with saddle-node transitions, crinkling transitions, tornadoes, and multicritical points.

At the side boundaries, the stable curve $s\left(\phi_{2}\right)$ remains smooth, and appears to coalesce with the unstable curve $u\left(\phi_{2}\right)$. At $b=0$ this is just the

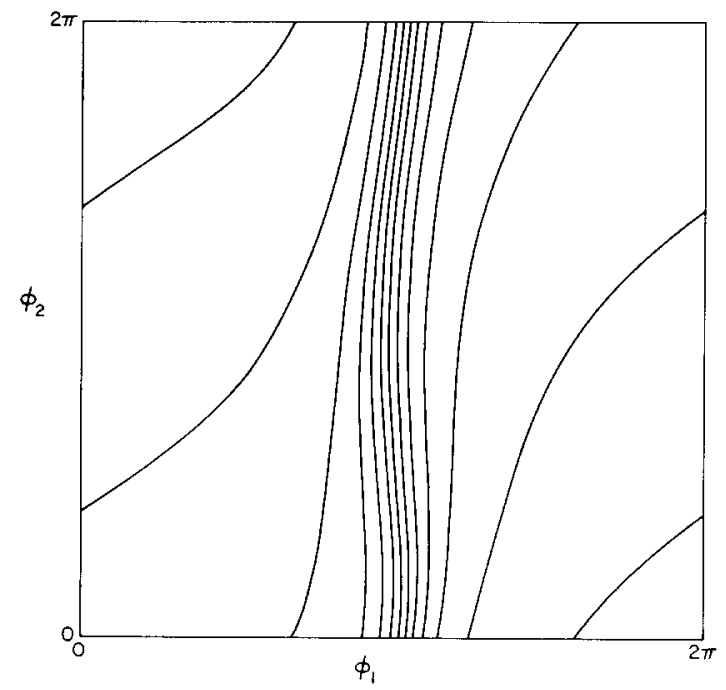

Fig. 3. Stable curve for another mode-locked state. A modelocked state with $\rho_{1}=(2 / 11) \rho_{2}-(1 / 11)$ is found in $f$ at $a=0.5$, $b=0.2, \omega=0.52\left(\rho_{2}=\sigma=(\sqrt{5}-1) / 2\right)$. It has a negative Liapunov exponent $\boldsymbol{A} \approx-0.00357$ and the winding number $\rho_{1}$ is constant under small changes in $a, b$ and $\omega$. One would expect reduced copies of fig. 2 about all mode locked states; we have seen several numerically. The mode-locked states are probably dense in parameter space, at least for small $a$ and $b$, but not of full measure. conventional saddle-node bifurcation of the circle map at $|a|=|\omega|$, since $f_{1}$ is then independent of $\phi_{2}$. As in the saddle-node bifurcation [6], on approaching the boundary at constant $b \neq 0$ from the mode-locked side the contraction rate (eq. (2.4)) $\Lambda \sim\left|\omega_{\mathrm{c}}-\omega\right|^{1 / 2}$. On the far side $\rho_{1} \neq 0$; the orbits spend most of their time running along the path of the former curves $s$ and $u$ (which no longer exist), with occasional rapid excursions that wrap around the torus once in the $\phi_{1}$ direction. The frequencies of these intermittent bursts determine the winding number $\rho_{1}$; as in the saddle-node bifurcation $\rho_{1} \sim\left|\omega-\omega_{\mathrm{c}}\right|^{1 / 2}$. (The contraction rate is of course non-zero and $\rho_{1}$ is constant in the other mode locked regions, as in fig. 3 )

A second way of displaying the saddle-node transition is by approximating [12] the irrational frequency ratio $\rho_{2}$ by a rational approximant $\sigma=p / q$. This will be a useful tool in studying all the transitions. The stable curve $s\left(\phi_{2}\right)$ becomes a stable $q$-cycle; iterating the map makes $s\left(\phi_{2}\right)$ a stable fixed point of $\left(f^{q}\right)_{1}\left(\phi_{1}, \phi_{2}\right)$ (by this notation we mean the first component of the $q$ th iterate of

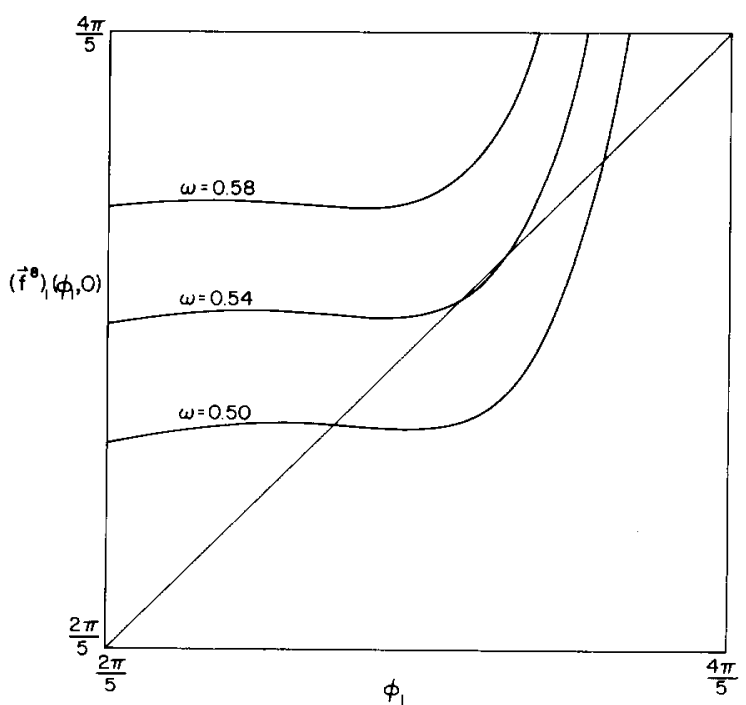

Fig. 4. Saddle-node transition: rational approximant. $\left(f^{8}\right)_{1}\left(\phi_{1}, 0\right)$ vs. $\phi_{1}$ for $\sigma=5 / 8, a=0.5, b=0.9$ and $\omega=0.50$, 0.54 and 0.58 . The saddle-node line in fig. 2 for $\sigma=(\sqrt{ } 5-1) / 2$ is a conventional saddle-node bifurcation of a stable and unstable $q$-cycle for rational approximants $p / q \sim \sigma$. 
the map). $\left(f^{q}\right)_{1}$ for $\phi_{2} \in[0,2 \pi / q]$ is analytically conjugate to $\left(f^{q}\right)_{1}$ for $\phi_{2} \in[2 \pi n / q, 2 \pi(n+1) / q]$; in this case we expect $f^{q}$ to be qualitatively $\phi_{2}$ independent. (See, however, the rational approximants at the multicritical point in 2.4.) We therefore can examine an essentially one-dimensional map $\left(f^{q}\right)_{1}$ $\left(\phi_{1}, \phi_{2}\right)$ versus $\phi_{1}$ (fig. 4). In essence we have "integrated out" the $\phi_{2}$ degree of freedom and all interesting physics occur in the $\phi_{1}$ direction. Here the one-dimensional map exhibits a saddle-node bifurcation, as expected.

Finally, it should be noted that although the saddle-node line extends into the region $|b|>1-|a|$ where $f$ is not invertible, the curve $s\left(\phi_{2}\right)$ does not intersect the singular curve $F$ in the $\left(\phi_{1}, \phi_{2}\right)$ plane at the bifurcation. Along the curve $F$ the contraction rate in the $\phi_{1}$ direction is infinite; at the transition the unstable curve $u\left(\phi_{2}\right)$ coincides with $s\left(\phi_{2}\right)$. It indeed seems reasonable (although not certain) that an unstable fixed curve will avoid regions of infinite contraction.

\subsection{The crinkling transition and tornadoes}

When the top boundary of the mode locked region in fig. 2 is crossed, the attractor appears to chaotically fill a two-dimensional volume, but does not wind in the $\phi_{1}$ direction ( $\rho_{1}$ stays zero). (A jump in the dimension of the attractor from one to two would doubtless be due to the form of $f_{2}$ in (1.1). Our observation is in accord with the Kaplan-Yorke conjecture [13] since when $\Lambda=0$ we have two Liapunov exponents $\geqslant 1$ ). The stable curves becomes fuzzy, turning into the tornado shown in fig. 5. At the transition, the map $f$ is always noninvertible and the curve $s\left(\phi_{2}\right)$. always intersects the curve of zero Jacobian $F$. The internal structures seen in the tornado are formed by the folding ( $f$ folds over the interior of $F$; the curve $F$ is the crease where $f$ is singular); one can also think of them as caustics in the projection of a higher dimensional attractor (with invertible dynamics) onto the torus.

Tornado attractors with winding number $\rho_{1}=0$ occur in the region shown in fig. 2 . At the bound-

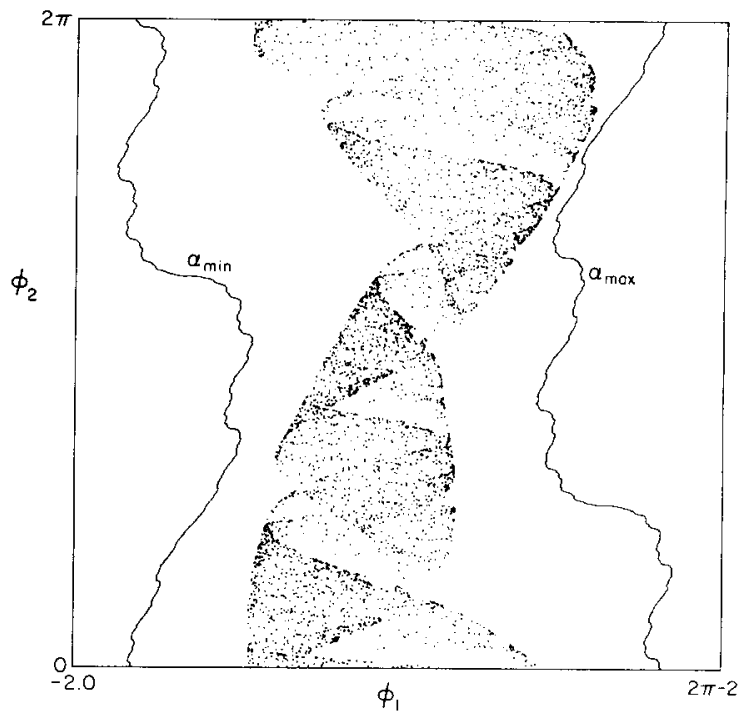

Fig. 5. Tornado with bounds. Attractor for $f$ with $a=0.5$, $b=1.4$ and $\omega=0$ exhibits chaotic motion about former stable curve. Side curves are rough bounds on the attractor found by comparing the dynamics to that of $\boldsymbol{f}$ with $a=0, b=1$, and $\rho_{1}=0(\omega=0.071458)$; they are the curves $\xi_{\alpha}$ for $x_{\min }=-1.557$ and $\alpha_{\max }=2.52$ in section 2.3. For smaller ' $a$ ' averaging over several iterates, with $b<1$, will give better bounds.

aries of this region, the tornadoes become intermittent; intermittent bursts cause repeated excursions of $\phi_{1}$ through $2 \pi$ in one direction. Crude numerical checks indicate the intermittency exponent (the power of $\left(\omega-\omega_{\mathrm{c}}\right)$ which gives the frequency of these bursts) is somewhat less than $1 / 2$. Above the intersection of the two boundaries, the bursts cause excursions of $\phi_{1}$ through $2 \pi$ in both directions, and $\rho_{1}$ probably depends upon initial conditions.

The confinement of the dynamics to a neighborhood of the former attracting curve in the "tornado" region is an interesting question; it can be understood by investigating the limit $a \rightarrow 0$. At $a=0$, the time evolution $f$ in eq. (1.1) depends only upon $\theta=\phi_{2}-\phi_{1}$, and reduces in this limit to the well-studied circle map $[2,3,9]$,

$g(\theta)=\theta+\bar{\omega}+b \cos \theta$.

The mode-locked region in fig. 2 collapses to the line $\bar{\omega}(b)$ on which the rotation number of the map is the golden mean $\sigma$; the crinkling transition line, 
the two multicritical points ( $M_{1}$ and $M_{2}$ in fig. 2) and the entire tornado region collapses onto the critical point $b=1$ where the circle map develops a cubic inflection point. Since $f$ depends only upon $\phi_{2}-\phi_{1}$, for fixed values of the constants $\boldsymbol{p}=(0, b, \bar{\omega}(b))$ one has a one-parameter family of invariant curves $\xi_{\alpha}$

$f_{1}\left(\xi_{\alpha}\left(\phi_{2}\right)\right)=\xi_{\alpha}\left(\phi_{2}+2 \pi \sigma\right)$

$\xi_{\alpha}\left(\phi_{2}\right)=\xi_{0}\left(\phi_{2}-\alpha\right)+\alpha$,

$\xi_{\alpha}(\alpha)=\alpha$.

For $b<1, \xi$ is analytic (so long as $\sigma$ is a good irrational); as $b \rightarrow 1, \xi$ becomes nondifferentiable. $\left(\xi_{0}\left(\phi_{2}\right)=h\left(\phi_{2}\right)-\phi_{2}\right.$, where $h$ is the coordinate transformation conjugating [2] the circle map $g$ to a simple rotation: $g \cdot h(\theta)=h(\theta+2 \pi \sigma)$.)

Under small deviations $\delta p=(a, \delta b, \delta \omega)$, points on $\xi_{\alpha}$ are mapped nearly back onto it;

$$
\begin{aligned}
& f_{1}^{p+\delta p}\left(\xi_{\alpha}\left(\phi_{2}\right), \phi_{2}\right)=\xi_{\alpha}\left(\phi_{2}+2 \pi \sigma\right)+\delta \omega \\
& \quad+a \cos \left(\xi_{\alpha}\left(\phi_{2}\right)\right)+\delta b \cos \left(\xi_{\alpha}\left(\phi_{2}\right)-\phi_{2}\right),
\end{aligned}
$$

with a slow drift to one side. We want to change variables from $\left(\phi_{1}, \phi_{2}\right)$ to $\left(\alpha, \phi_{2}\right)$ with $\alpha$ defined such that $\xi_{\alpha}\left(\phi_{2}\right)=\phi_{1}$ (since each point $\left(\phi_{1}, \phi_{2}\right)$ lies on an invariant curve, this variable change is well defined and for $b<1$ is analytic). We can then use $\alpha$ as a slow variable. The change $\delta \alpha\left(\alpha, \phi_{2}\right)$ in $\alpha$ under one iteration of $f^{p+\delta_{p}}$ is defined implicitly by

$\xi_{\alpha+\delta \alpha}\left(\phi_{2}+2 \pi \sigma\right)=f_{1}^{p+\delta p}\left(\xi_{\alpha}\left(\phi_{2}\right), \phi_{2}\right)$.

Using eq. (2.7), for $b<1$ to first order in $\delta p$ we can solve for $\delta \alpha$,

$$
\begin{aligned}
(\delta \alpha / a)= & {\left[(\delta \omega / a)+\cos \left(\xi_{\alpha}\left(\phi_{2}\right)\right)\right.} \\
& \left.+(\delta b / a) \cos \left(\xi_{\alpha}\left(\phi_{2}\right)-\phi_{2}\right)\right] \\
& /\left[1-\left(\partial \xi_{\alpha} / \partial \phi_{2}\right)\left(\phi_{2}+2 \pi \sigma\right)\right] .
\end{aligned}
$$

If we let $t$ be the number of iterations of the map, the averaged equation of motion for $\alpha$ becomes

$$
\begin{aligned}
\frac{1}{a} \frac{\mathrm{d} \alpha}{\mathrm{d} t}= & \int_{0}^{2 \pi} \frac{\mathrm{d} \phi_{2}}{2 \pi}\left[(\delta \omega / a)+\cos \left(\xi_{\alpha}\left(\phi_{2}\right)\right)\right. \\
& \left.+(\delta b / a) \cos \left(\xi_{\alpha}\left(\phi_{2}\right)-\phi_{2}\right)\right] \\
& /\left[1-\frac{\partial \xi_{\alpha}}{\partial \phi_{2}}\left(\phi_{2}+2 \pi \sigma\right)\right] .
\end{aligned}
$$

This equation roughly corresponds to the amplitude equations used in studying convection. It ignores the fast timescales (i.e., $\phi_{2}$ ). One can extract from it most of the features of the phase diagram even at $a=1 / 2$ (fig. 2). We have evaluated the right-hand side of eq. (2.11) numerically as a function of $\alpha$. Crudely speaking, $\xi_{\alpha}\left(\phi_{2}\right) \sim \alpha$, and the middle cosine term in the numerator of (2.11) gives a periodic potential $\mathrm{d} \alpha / \mathrm{d} t$ proportional to $\cos (\alpha)$, with one minimum near $\alpha=\pi / 2$. (As $b \rightarrow 1$ the amplitude of this potential diverges, since the denominator goes to zero at a dense set of points, but the form remains the same.) The first term involving $\delta \omega$ tilts this potential; $\mathrm{d} \alpha / \mathrm{d} t \sim$ $\cos \alpha+\delta \omega / a$. Until $\delta \omega$ becomes comparable to $a$, it can only shift the minimum and cannot cause $\phi_{1}$ to wind. The perturbations contributed by $\delta b$ similarly can cause intermittency only when $\delta b \gtrsim a$. Thus the region of zero $\phi_{1}$ winding number in fig. 2 is roughly of radius $a$ about the curve $\bar{\omega}(b)$ of the circle map.

However, the averaged equation completely misses the crinkling transition. The fast timescale corrections to eq. (2.11) in the mode locked region act to deform the curve $\xi\left(\phi_{2}\right)$; in the tornado region they destroy it, introducing chaos. By using eq. (2.10), one can put rough bounds on the extent of the chaotic attractor; fig. 5 shows the bounds given by finding $\alpha_{\min }<\alpha_{\max }$ such that $\delta \alpha \geqslant 0$ for all points $\xi_{\alpha \min }\left(\phi_{2}\right)$ and $\delta \alpha \leqslant 0$ for all points $\xi_{\alpha \max }\left(\phi_{2}\right)$, at $b=1$. (Strict bounds on the attractor can also be found.) Better bounds for small $a$ can be found by iterating eq. (2.10), until as $a \rightarrow 0$ the asymptotic phase diagram should be given by eq. (2.11).

It is not widely appreciated that averaged equa- 
tions and amplitude equations cannot exclude small-scale noise on fast timescales. For good winding numbers, if $A<0$ the stable invariant curve must persist for small variations in the parameters (section 2.1) and hence one can exclude chaos. In contrast, the absence of chaos cannot be inferred from the averaged equations. The latter do on the other hand serve to confine the chaos (e.g., prove $\rho_{1}$ stays zero).

At the top boundary of fig. 2, the curve $s\left(\phi_{2}\right)$ becomes nonanalytic and appears to develop horizontal tangents at a dense set of points (fig. 6). The map $f$ in eq. (1.1) has zero Jacobian on the football-shaped curve $F$ in fig. 6; the curve $s\left(\phi_{2}\right)$ crosses $F$ several times. The contraction rate $\Lambda$ goes to zero as one approaches the crinkling transition from below (fig. 7), but in an irregular fashion. Presumably the dips in fig. 7 occur when a new intersection of $s$ with $F$ forms, and $s$ is temporarily tangent to $F$. The time series at long times will depend upon the interplay of influences of these intersections; the distance separating them in the $\phi_{2}$ direction will be relevent parameters. The power

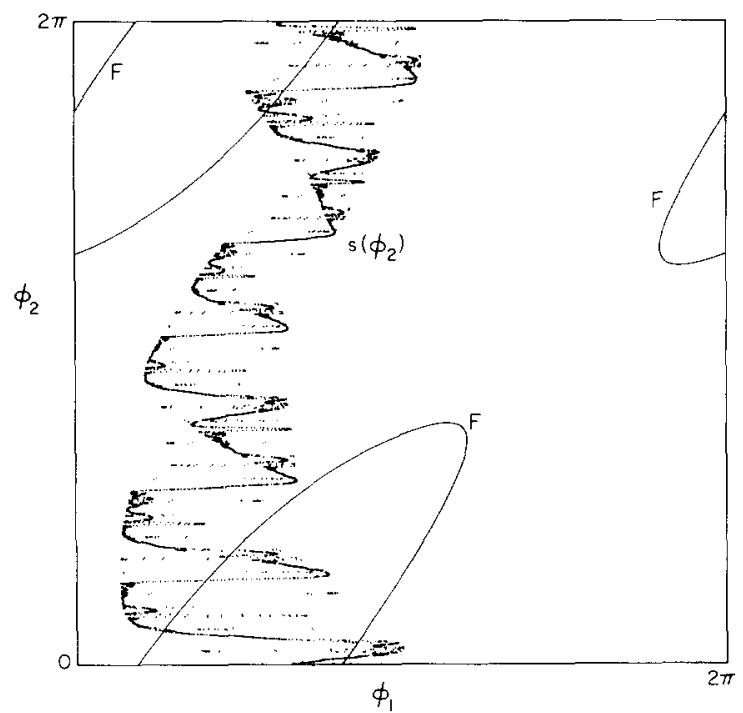

Fig. 6. Stable curve at crinkling transition. Attracting curve $s$ for eq. (1.1) with $a=0.5, b=1.3$ and $\omega=0.2$, near where $s$ disappears in a crinkling transition. The $\phi_{2}$ coordinates of the points at which $s$ crosses the zero Jacobian line $F$ are probably relevant in determining its singular structure.

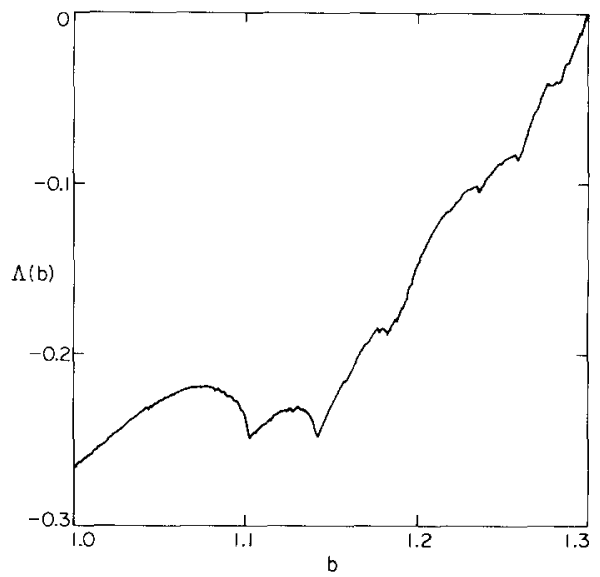

Fig. 7. Contraction rate at the crinkling transition. Contraction rate $A$ (eq. (2.4)) as a function of $b$ for $\omega=0.0, a=0.5$. The crinkling transition occurs at $b_{\mathrm{c}}=1.299 \pm 0.001$. The error bars on $b_{\mathrm{c}}$ are perhaps overly conservative. The periodic cycle of length 17711 (corresponding to the 22nd rational approximant $p_{22} / q_{22}$ to $\sigma$ ) becomes unstable at $b=1.29893 \pm 1$ if the cycle is started at $\phi_{2}=0$; it becomes unstable at $b=1.299428 \pm 10$ if the cycle is started at $\phi_{2}=2 \pi / 10$. Thus the 17711 cycle has a transition in a range $b=1.2992 \pm 0.0003$. However, the convergence of $A$ with increasing cycle length is very slow; at $b=1.298$ it has clearly not yet converged $\left(A_{17711}=-5.85 \times 10^{-4}, A_{46368}=-14.07 \times 10^{-4}\right)$. Major features seen here are independent of cycle length, but the very small scale bumps are finite cycle-length effects. (For $b$ in the range $1.2-1.3$ cycles of length 46368 were used.)

spectrum at the crinkling transition has been examined numerically at isolated points on $M_{1} M_{2}$ (fig. 8 ); it is not self-similar but is singular in that the envelope of the spectrum varies as frequency $v$ to a power as $v \rightarrow 0$. Thus scaling behavior does not appear to occur unless these parameters (and possibly others) are controlled.

Finally, we can again let $\sigma$ be a rational approximant $p / q$ of the golden mean; fig. 9 shows that, at the transition, $\left(f^{4}\right)_{1}$ is developing multiple fixed points, via an inverse saddle-node bifurcation. This is the first stage in a complicated transition also including period doubling, which as $q \rightarrow \infty$ collapses onto the crinkling transition.

\subsection{The multicritical point}

The saddle-node line and the crinkling line terminate at a common multicritical point. New 


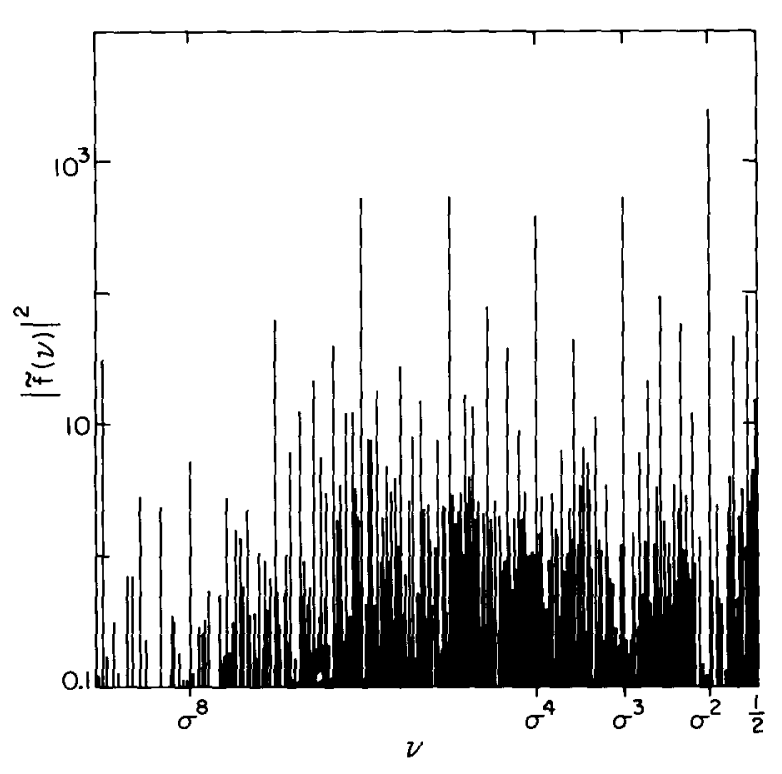

Fig. 8. Time series spectrum at the crinkling transition. Power spectrum of the time series $\phi_{1}^{(n)}=\left(f^{n}\left(\phi_{1}^{0}, \phi_{2}^{0}\right)\right)_{1}$ for $f$ in eq. (1.1) at the crinkling transition $\left(a=\frac{1}{2}, \omega=0, b=1.298926\right.$, $\left.\sigma=2 \pi(10946 / 17711), \phi_{1}^{0}=-0.0364373, \phi_{2}^{0}=0\right)$, on a $\log -\log$ plot. The largest peaks fall at $v=\sigma^{j}=[(\sqrt{5}-1) / 2]^{j}$. Note that no factor has been divided out of this spectrum (in contrast to fig. 11); the low frequency behavior at the crinkling transition probably is more singular than at the multicritical point.

critical exponents appear at this point. As one crosses the saddle-node line just below it, fig. 10 shows the crossover of the contraction exponent from a multicritical value of $\approx 1 / 3$ (found by fitting the slope) to its saddle-node value of $1 / 2$. Also, the time series spectrum $f_{1}(v)$ at the multicritical point becomes self-similar at low frequencies (fig. 11). The spectrum appears to follow the scaling law (fig. 12)

$\tilde{f}_{1}(v)=\sigma^{2} \tilde{f}_{1}\left(v / \sigma^{2}\right)$, as $v \rightarrow 0$

For $a=0$, the scaling law follows from that of the circle map [2] $(a=0, b=1, \bar{\omega}=0.071458 \ldots)$ if the latter is iterated twice. Presumably the relative phase (e.g., the angle in fig. 12) of the even and odd peaks $\tilde{f}\left(v / \sigma^{2 n}\right)$ and $\tilde{f}\left(v / \sigma^{2 n+1}\right)$ goes smoothly to $180^{\circ}$ as $a \rightarrow 0$.

As one increases $\omega$ along the crinkling transition line, eventually the singular attracting curve $s$ in

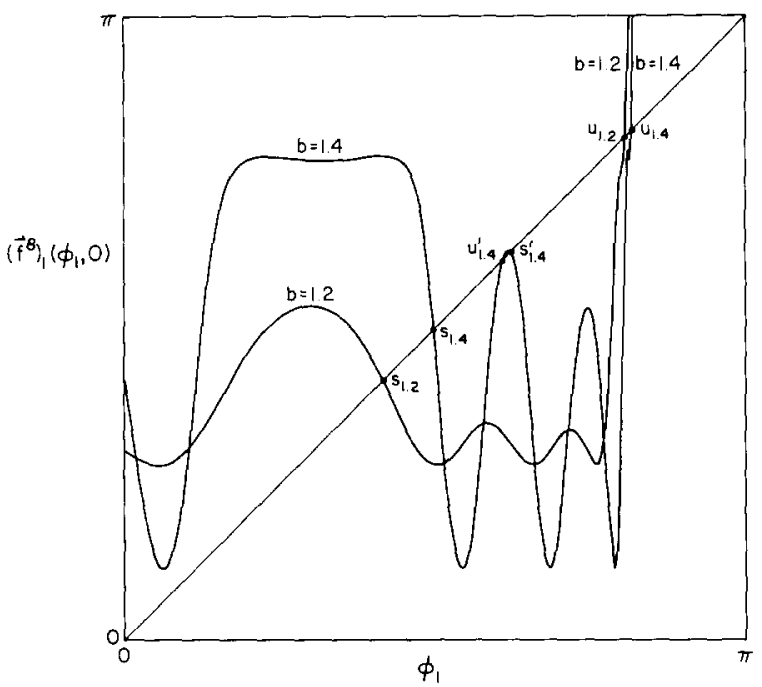

Fig. 9. Crinkling transition: rational approximants. $\left(f^{8}\right)_{1}\left(\phi_{1}, 0\right)$ vs. $\phi_{1}$ for $\sigma=5 / 8$, as $a=0.5, \omega=0.4$ and $b=1.2$ and 1.4. The crinkling bifurcation occurs when multiple zeroes form in the rational approximants, via inverse saddle-node bifurcations. For $b=1.2$ (representing the mode locked state) there is a single stable fixed point $s_{1.2}$ and a single unstable point $u_{1.2}$ corresponding to $s$ and $u$ of fig. 1 . A second pair of fixed points $u_{1.4}^{\prime}$, $s_{1.4}^{\prime}$ has formed at $b=1.4$.

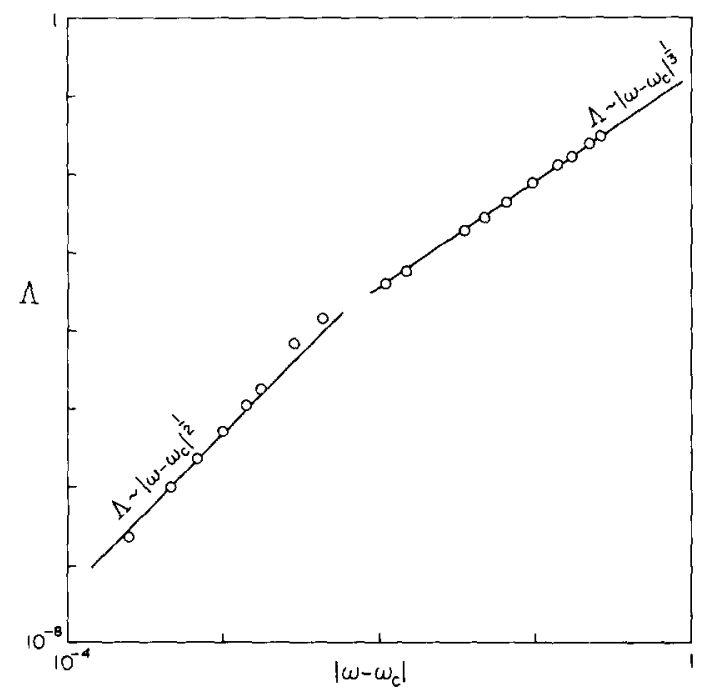

Fig. 10. Crossover in $A$ from multicritical to saddle-node scaling behavior along $b=1.0$. The contraction rate $A$ in eq. (2.4) scales as $A \propto \mid \omega-\omega_{c}^{1 / 2}$ as one approaches the saddlenode transition at fixed $b$ from the mode locked side. At the multicritical point $\mathbf{M}_{2}$ (see fig. 2$)(b \approx 1.00245, \omega \approx 0.529192)$ $A$ presumably scales with a new multicritical exponent (eq. (3.12)). Here we see a crossover from multicritical scaling to saddle-node scaling as we cross the saddle-node line at $b=1.0$, $\omega_{c}=0.52921796$ just below $\mathbf{M}_{2}$. 


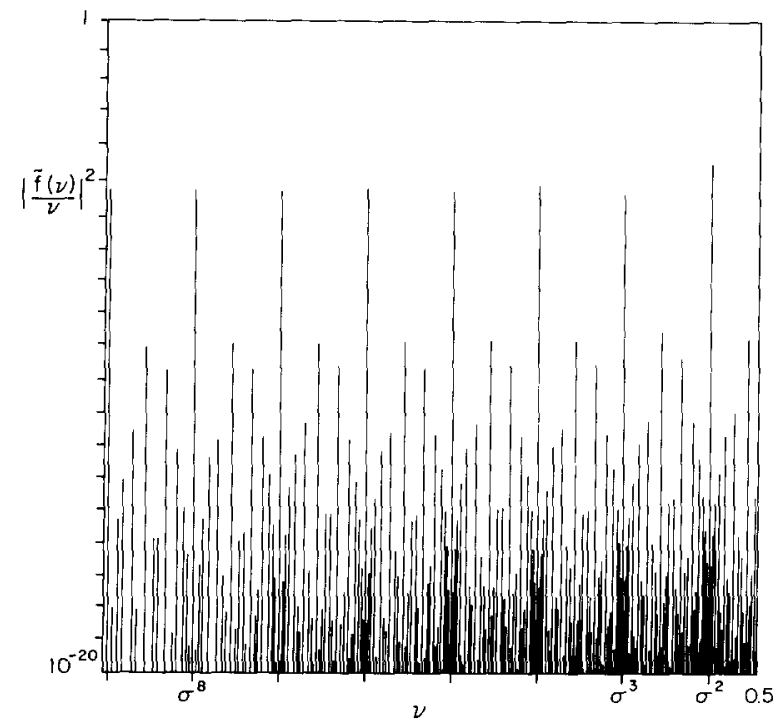

Fig. 11. Time series power spectrum at multicritical point. Power spectrum of the time series $\phi_{1}^{(n)}=\left(f^{n}\left(\phi_{1 \mathrm{c}}, \phi_{2 \mathrm{c}}\right)\right)_{1}$ for $f$ in eq. (1.1) at the multicritical point $\mathrm{M}_{2} \quad(b=1.00245$, $\omega=0.529192)$. A normalization factor of $v^{2}$ has been divided out of the power, and a log-log plot has been used, in order to exhibit the scaling implied by eq. (2.12). The principle peaks fall at $v=\sigma^{j}=[(\sqrt{ } 5-1) / 2\}^{j}$. Except for an overall scale factor, the spectrum should be universal as $v \rightarrow 0$.

fig. 6 becomes tangent to the zero Jacobian curve $F\left(\partial f_{1} / \partial \phi_{1}=0\right)$ of the map (fig. 13). The point of tangency is the extremal point $\left(\phi_{1}^{\mathrm{c}}, \phi_{2}^{\mathrm{c}}\right)$ of $F$ in the $\phi_{2}$ direction. Since the crinkling transition depends upon the noninvertibility of $f$, the crinkling transition line must end when $s$ is tangent to $F$, and indeed this is the multicritical point. There is now a unique intersection point about which to scale; we shall argue in section 3 that this explains the self-similar time series spectrum.

The fact that at the multicritical point $s$ and $F$ are tangent at $\left(\phi_{1}^{\mathrm{c}}, \phi_{2}^{\mathrm{c}}\right)$ can be explained as a consequence of the coexistence of the saddle-node and crinkling transitions and of zero winding number $\rho_{1}$ in the $\phi_{1}$ direction. The map $f_{1}\left(\phi_{1}, \phi_{2}\right)$ as a function of $\phi_{1}$ is monotone increasing outside $F$, and monotone decreasing inside $F$. ( $f$ folds the interior over, with $F$ forming the crease.) If $u$ were tangent to $F$ at a single point other than at an extremum, parts of the interior of $F$ would neces-

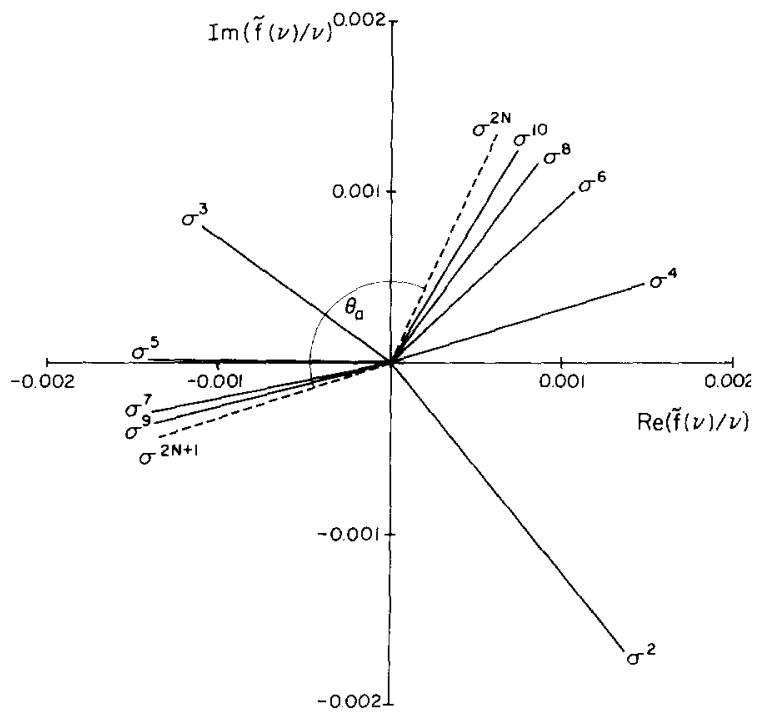

Fig. 12. Scaling of the time series spectrum at the multicritical point. The principle peaks of fig. 11 , here plotted in the complex plane. The even peaks $v=\sigma^{2 n}$ and the odd peaks $v=\sigma^{2 n+1}$ appear to be separately converging to universal values marked $\sigma^{2 N}$ and $\sigma^{2 N+1}$ as $n \rightarrow \infty$. At $a=0$ (the circle map) the fourier spectrum at low frequencies satisfies $\tilde{f}(v)=-\sigma \widetilde{f}(v / \sigma)$; thus the opening angle $\theta_{a}$ is $\pi$ at $a=0$. The time series used was of length 2584 , so the principle peaks continue down to $1 / 2584=\sigma^{16}$. Below $\sigma^{10}$ neither the magnitudes nor the phases of the fourier peaks scale; this is presumably due to the cutoff at $\sigma^{16}$, with possibly some contribution from errors in the value of $b$ at the multicritical point.

sarily be mapped across $u$. The onset of intermittency necessarily involves orbits which cross the unstable manifold $u$. We believe near the multicritical point the converse is also true; points crossing $u$ when $u$ is tangent to $F$ won't be mapped back across (roughly because outside $F$ the map is increasing). Under further iterations, these points will presumably wind around in $\phi_{1}$, some of them reentering $F$ and crossing again - leading to nonzero $\rho_{1}$. Thus for $u$ to be tangent to $F$ at any point other than its extreme in the $\phi_{2}$ direction, intermittency must already be present. For a crinkling transition to take place, $s$ must intersect $F$; at a saddle-node transition, $s$ and $u$ coalesce; $u$ will presumably not cross $F$ (as noted in 2.2). Thus $u$ and $s$ must be tangent to $F$, and for $\rho_{1}$ to stay zero the point of tangency must be an extremum of $F$ 


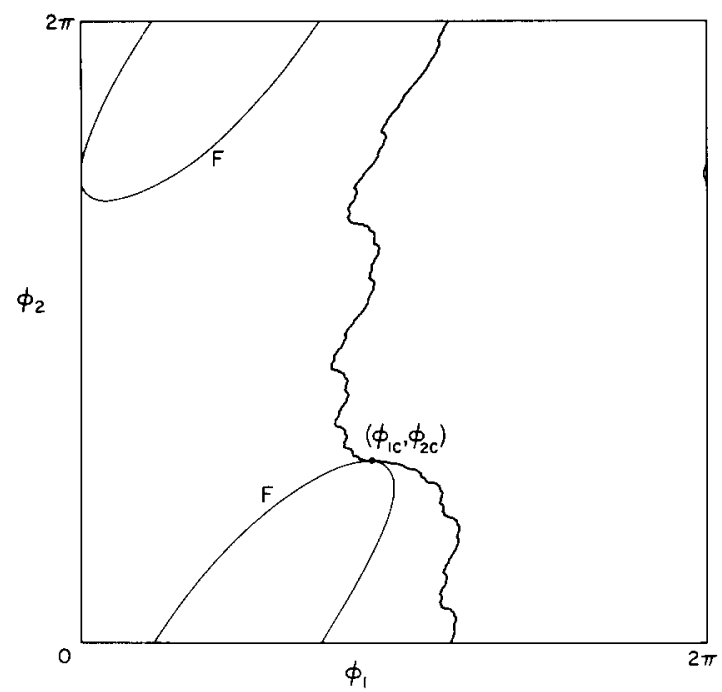

Fig. 13. Multicritical curve. At the multicritical point $\mathbf{M}_{2}$, the stable and unstable curves of eq. (1.1) coalesce into the curve shown here (whose time series spectrum is shown in figs. 11 and 12 ). The curve is tangent to the zero Jacobian curve $F$ (where $f$ does not locally have an inverse) at the point $\left(\phi_{1 \mathrm{c}}, \phi_{2 c}\right)=(2.894,1.828)$ on $F$ where $\phi_{2}$ is a local extremum. The tangency follows from the simultaneous occurrance of a saddle-node and a crinkling bifurcation; the unstable curve $u$ does not cross $F$ at saddle-node transitions, (fig. 1) while the stable curve $s$ always crosses $F$ at crinkling transitions (fig. 6). The tangency must occur at the extremum in $\phi_{2}$ to maintain the winding number $\rho_{1}=0$.

in the $\phi_{2}$ direction, where $f\left(\phi_{1}, \phi_{2}\right)$ as a function of $\phi_{1}$ has a cubic inflection point.

Fig. 14 shows $\left(f^{q}\right)_{1}\left(\phi_{1}, \phi_{2}\right)$ versus $\phi_{1}$, at the multicritical point, with $\sigma=p / q$ approximately the inverse golden mean. We can understand how the saddle-node and crinkling transitions manifest themselves at the multicritical point by looking at this function at various values of $\phi_{2}$ in an interval $1 / q$. (The qualitative features then of course repeat.)

We start with the curve labeled zero, in which $\phi_{2}=\phi_{2}^{\mathrm{c}}$ (the local maximum of $F$ in the $\phi_{2}$ direc-

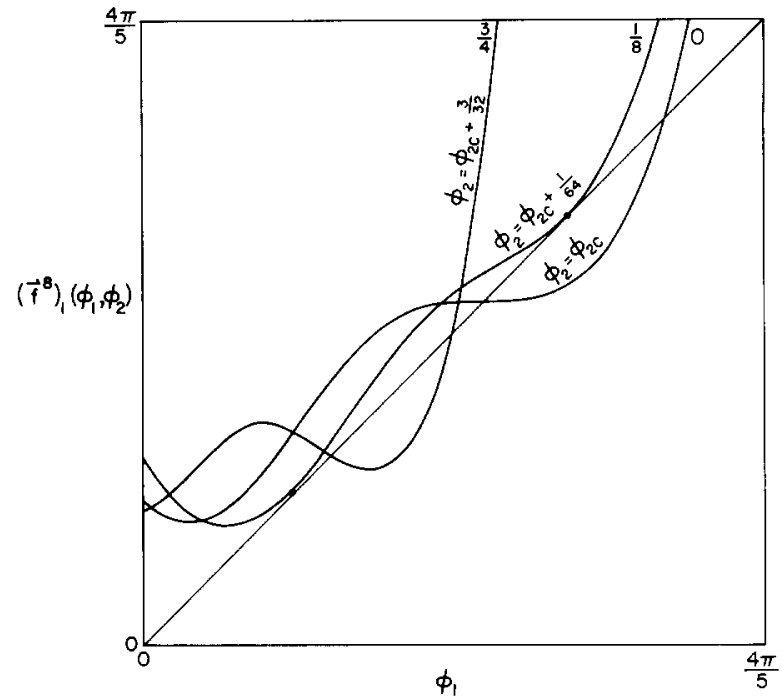

Fig. 14. Multicritical point: rational approximants. $\left(f^{8}\right)_{1}\left(\phi_{1}, \phi_{2}\right)$ vs. $\phi_{1}$ at the multicritical point for $\sigma=5 / 8$ and $a=0.5$, at $\phi_{2}=\phi_{2 c}, \phi_{2 c}+1 / 64$ and $\phi_{2 c}+3 / 32$. At $\phi_{2}=\phi_{2 c}+1 / 8$, the curve would again have a cubic inflection fixed point at a smaller value of $\phi_{1}$. One can see clearly the coexistence of the saddlenode and crinkling bifurcations in the curve $\phi_{2 c}+1 / 64$, one eighth the way through the cycle. Here the saddle-node bifurcation is occurring apparently at the same time as a new pair of fixed points (signaling a crinkling bifurcation) is forming via an inverse saddle-node bifurcation.

tion); the iterated map $f^{q}$ thus has a cubic inflection point at $\phi_{1}^{\mathrm{c}}$. The function here has a single stable fixed point at $s_{0}$ and a single unstable fixed point at $u_{0}$. The curve labeled $1 / 8$ shows $\left(f^{q}\right)_{1}$ at $\phi_{2}=\phi_{2}^{\mathrm{c}}+1 / 8 q ; s_{0}$ and $u_{0}$ have just annihilated in a saddle-node bifurcation. However, there has been an inverse saddle-node bifurcation (characteristic of the rational approximants at the crinkling transition) at precisely the same time. The curves continue to evolve until at $\phi_{2}^{\mathrm{c}}+1 / q$ a cubic inflection point again appears between the new stable and unstable points.

\section{Proposed renormalization group}

In this section we propose, but do not implement, a renormalization-group analysis of nonlinear systems forced at two frequencies (eq. (1.1)). We study three known fixed points of this renormalization group, and 


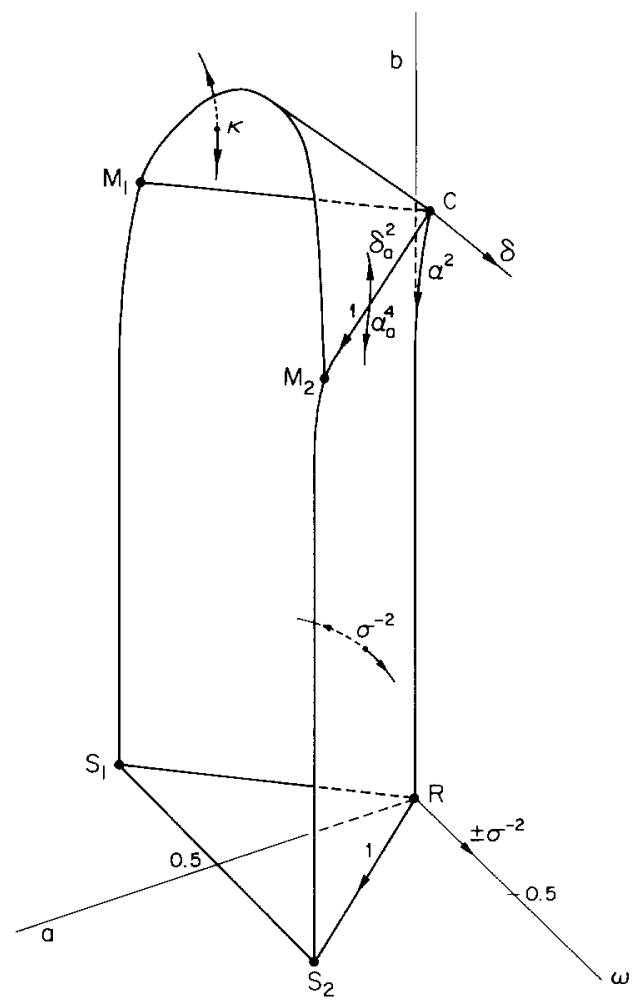

Fig. 15. Phase diagram and renormalization group eigenvalues. Inside the volume $\mathbf{M}_{1} \mathbf{M}_{2} \mathrm{~S}_{2} \mathrm{~S}_{1} \mathrm{RC}$ eq. (1.1) has a smooth attractor $\phi_{1}=s\left(\phi_{2}\right)$ with winding number $\rho_{1}=0$ in the $\phi_{1}$ direction. The eigenvalues shown govern the various bifurcations through which this mode locked state can break down. Point $\mathrm{R}$ is the trivial fixed point; the curve connecting $\mathrm{C}$ to $\mathrm{R}$ is the intersection of the stable manifold of $\mathrm{R}$ with $[a, b, \omega]$. The surfaces $\mathrm{M}_{1} \mathrm{CRS}_{1}$ and $\mathrm{M}_{2} \mathrm{CRS}_{2}$ are the intersection of $[a, b, \omega]$ with the stable manifold of the saddle-node fixed points; the eigenvalue governing the penetration of these surfaces is $\sigma^{2}$, so the exponent is $\log \sigma / \log \left(\sigma^{-2}\right)=-1 / 2$. The point $\mathrm{C}$ lies on the stable manifold of the circle map fixed point. The eigenvalue $\delta$ governs perturbations depending only on $\theta_{2}-\theta_{1}$ causing transitions to different winding numbers $\rho_{1}$; the eigenvalue $\alpha^{2}$ governs the growth of perturbations depending on $\theta_{2}-\theta_{1}$ at fixed winding number. The multicritical lines $\mathbf{M}_{1} \mathrm{C}$ and $\mathrm{M}_{2} \mathrm{C}$ are conjectured to be the intersection of $[a, b, \omega]$ with the stable manifold of a line of multicritical fixed points of $T^{2}$. The conjectured eigenvalues $\alpha_{a}^{4}$ and $\delta_{a}^{2}$ of $T^{2}$ at these multicritical fixed points thus lead to exponents $\log \sigma^{2} / \log \alpha_{a}^{4}$ and $\log \sigma^{2} / \log \delta_{a}^{2}$ governing perturbations leading down the saddle-node surface and across the crinkling surface, respectively. Finally, althought the crinkling surface intersecting $[a, b, \omega]$ in $\mathbf{M}_{1} \mathbf{C M}_{2}$ is probably not a stable manifold of a fixed point, it is mapped into itself under $T$. If the motion on this codimension one surface is "sufficiently ergodic", one might characterize flow away from it by an eigenvalue $\kappa$.

relate them to the phase diagram (fig. 15). We conjecture that a one-parameter family of multicritical fixed points exists. Finally, we briefly speculate about possible studies of the crinkling transition.

The non-trivial universal features associated with transitions in dynamical systems always involve behavior on long timescales. The usual method for extracting these universal features is to decimate the time series. Since the time series is one-dimensional, this decimation is given exactly, by iterating the map.

For convenience, we shall write our renormalization group in terms of variables $\theta_{1}$ and $\theta_{2}$ periodic modulo one; the renormalization group transformation will iterate the map and expand about $\theta_{1}=\theta_{2}=0$. (The expansion point in terms of the $2 \pi$-periodic variables $\phi_{1}$ and $\phi_{2}$ will be shifted appropriately as we study different transitions.) We express $f\left(\theta_{1}, \theta_{2}\right)=\left(f_{1}\left(\theta_{1}, \theta_{2}\right),\left(\theta_{2}+\sigma\right)\right)$ in terms of two pieces, $\boldsymbol{E}$ and $\boldsymbol{F}$, whose 
second components are trivial:

$$
\boldsymbol{f}\left(\theta_{1}, \theta_{2}\right)=\left\{\begin{array}{c}
\boldsymbol{E}\left(\theta_{1}, \theta_{2}\right)=\left(\begin{array}{c}
E_{1}\left(\theta_{1}, \theta_{2}\right) \\
\theta_{2}+\sigma
\end{array}\right), \quad \sigma-1<\theta_{2}<0, \\
\boldsymbol{F}\left(\theta_{1}, \theta_{2}\right)=\left(\begin{array}{c}
F_{1}\left(\theta_{1}, \theta_{2}\right) \\
\theta_{2}+\sigma-1
\end{array}\right), \quad 0<\theta_{2}<\sigma .
\end{array}\right.
$$

Our renormalization group is a map $T$ on the space of pairs of functions $(\boldsymbol{E}, \boldsymbol{F})$. Consistent with the interpretation of $\theta_{2}$ as an external periodic force, we restrict the second components of $\boldsymbol{E}$ and $\boldsymbol{F}$ to be trivial (as in eq. (3.1)). We can also assume $\boldsymbol{E}$ and $\boldsymbol{F}$ commute; they of course commute initially if $f$ is analytic, and our renormalization group will preserve this property.

Following the treatment of annular maps in ref. 2, we define

$$
\begin{aligned}
& T(\boldsymbol{E}, \boldsymbol{F})=\left(A F A^{-1}, A F \cdot E A^{-1}\right), \\
& A=\left(\begin{array}{cc}
\alpha & \beta \\
0 & -\sigma^{-1}
\end{array}\right),
\end{aligned}
$$

where the choice of $\alpha$ and $\beta$ in $A$ will in general depend upon $\boldsymbol{E}$ and $\boldsymbol{F}$. This transformation preserves the winding number $\rho_{2}=\sigma=(\sqrt{ } 5-1) / 2$ the ratio of the two forcing frequencies. (Remember that this is a relevant variable; the low frequency properties of $f$ depend sensitively, for example, on whether $\rho_{2}$ is rational or irrational.) The best rational approximants to $\sigma$ are ratios $q_{n} / q_{n+1}$ of Fibonacci numbers $q_{0}=0, q_{1}=1$, $q_{n+1}=q_{n}+q_{n-1}$. After $n$ applications of $T, E^{(n)}=A^{n} f^{q_{n}} A^{-n}, F^{(n)}=A^{n} f^{q_{n+1}} A^{-n}$.

The contraction rate $A$ (eq. (2.4)) behaves simply under $T . \Lambda(f)$ is the integral of the logarithm of the magnitude of the Jacobian of $\boldsymbol{f}$ over its attracting curve,

$$
\Lambda(f)=\int_{\sigma-1}^{\sigma} \mathrm{d} \theta_{2} \log \left|\frac{\partial f_{1}}{\partial \theta_{1}}\left(s_{f}\left(\theta_{2}\right), \theta_{2}\right)\right| .
$$

$T$ acts by eliminating those elements of the time series which lie in the range $\sigma^{3}<\theta_{2}<\sigma$, and then scaling the coordinates by $A$. Thus the segment of the attracting curve $s_{f}\left(\theta_{2}\right)$ of $f$ lying between $-\sigma^{2}$ and $\sigma^{3}$ maps under $A$ onto the attracting curve $s_{T f}$ of $T f$ :

$$
s_{T f}\left(\theta_{2}\right)=\alpha s_{f}\left(-\sigma \theta_{2}\right)-\beta \sigma \theta_{2} .
$$

(In particular, $s_{T f}$ is continuous at the boundary only if one identifies the points $\boldsymbol{E}\left(\theta_{1}, 0\right)$ with $\boldsymbol{F}\left(\theta_{1}, 0\right)$ after applying $T$. When the map becomes non-invertible along $\theta_{2}=0$, the utility of gluing the torus back in this way becomes questionable.) The Jacobian of $T f$ along $s_{T f}$ for $-\sigma^{2}<\theta_{2}<0$ is equal to that of $f$ at the corresponding point on $s_{f}$ :

$$
\begin{aligned}
& T f\left(\theta_{1}, \theta_{2}\right)=\left(\begin{array}{c}
\alpha F_{1}\left(\alpha^{-1} \theta_{1}+\frac{\beta \sigma}{\alpha} \theta_{2},-\sigma \theta_{2}\right)+\beta\left(-\sigma \theta_{2}+\sigma-1\right) \\
\theta_{2}+\sigma
\end{array}\right), \quad-\sigma^{2}<\theta_{2}<0, \\
& \frac{\partial(T f)_{1}}{\partial \theta_{1}}\left(s_{Y y}\left(\theta_{2}\right), \theta_{2}\right)=\frac{\partial F_{1}}{\partial \theta_{1}}\left(s_{F}\left(-\sigma \theta_{2}\right),-\sigma \theta_{2}\right), \quad-\sigma^{2}<\theta_{2}<0 .
\end{aligned}
$$

The Jacobian of $T f$ along $s_{T f}$ in the range $0<\theta_{2}<\sigma$ is a product of the Jacobians for $f$ at the corresponding 
point on $s_{f}$ and at its image (which was eliminated by the decimation). That is, for $0<\theta<\sigma$,

$$
\begin{aligned}
& T f\left(\theta_{1}, \theta_{2}\right)=\left(\begin{array}{c}
\alpha F_{1}\left[E_{1}\left(\alpha^{-1} \theta_{1}+\frac{\beta \sigma}{\alpha} \theta_{2},-\sigma \theta_{2}\right),-\sigma \theta_{2}+\sigma\right]+\beta\left(-\sigma \theta_{2}+\sigma^{3}\right) \\
\theta_{2}+\sigma-1
\end{array}\right), \\
& \frac{\partial(T f)_{1}}{\partial \theta_{1}}\left(s_{T f}\left(\theta_{2}\right), \theta_{2}\right)=\frac{\partial F_{1}}{\partial \theta_{1}}\left(s_{f}\left(-\sigma \theta_{2}+\sigma\right),-\sigma \theta_{2}+\sigma\right) \frac{\partial E_{1}}{\partial \theta_{1}}\left(s_{f}\left(-\sigma \theta_{2}\right),-\sigma \theta_{2}\right), \quad 0<\theta_{2}<\sigma .
\end{aligned}
$$

Thus, using (3.4),

$$
\Lambda(f)=\sigma \Lambda(T f) \text {. }
$$

It is obvious from our definition of $T$ that, roughly speaking, after a large number of iterations $f^{N} \approx(T f)^{N}$ up to a scale change. Thus by taking the Jacobian of both sides, for large $N \mathrm{e}^{N A(f)} \approx \mathrm{e}^{N a A(T f)}$; hence (3.8)

If $B$ is a change of coordinates which commutes with $A$ and if $(E, F)$ is a fixed point of $T$, then so it $\left(B E B^{-1}, B F B^{-1}\right)$. We allow only changes of coordinates which leave the $\theta_{2}$ evolution trivial; thus if $B$ is linear, it has the form $\left(\begin{array}{c}+ \\ 0\end{array} \frac{h}{1}\right)$ and commutes with $A$ if $h\left(\alpha+\sigma^{-1}\right)=\beta g$. All of our fixed points lie withir one-parameter families generated by scaling them in this way as we shall see.

The first three entries in table I are known fixed points of $T$ whose winding number $\rho_{1}$ in the $\theta_{1}$ direction is zero. Also shown are the physically relevant known eigenvectors and eigenvalues of the linearized fixed point equations; if $\boldsymbol{E}$ and $\boldsymbol{F}$ are fixed points of $T$ then a small perturbation $E_{1}+\epsilon, F_{1}+\delta$ grows with eigenvalue $\lambda$ if

$$
\begin{aligned}
& \lambda c\left(\alpha \theta_{1}+\beta \theta_{2},-\sigma^{-1} \theta_{2}\right)=\alpha \delta\left(\theta_{1}, \theta_{2}\right), \\
& \lambda \delta\left(\alpha \theta_{1}+\beta \theta_{2},-\sigma^{-1} \theta_{2}\right)=\alpha \frac{\partial F_{1}}{\partial \theta_{1}}\left(E\left(\theta_{1}, \theta_{2}\right)\right) \epsilon\left(\theta_{1}, \theta_{2}\right)+\alpha \delta\left(E\left(\theta_{1}, \theta_{2}\right)\right) .
\end{aligned}
$$

In addition, the condition that $\boldsymbol{E}$ and $\boldsymbol{F}$ commute $(\boldsymbol{E} \cdot \boldsymbol{F} \equiv \boldsymbol{F} \cdot \boldsymbol{E})$ implies

$$
\frac{\partial F_{1}}{\partial \theta_{1}}\left(\boldsymbol{E}\left(\theta_{1}, \theta_{2}\right)\right) \epsilon\left(\theta_{1}, \theta_{2}\right)+\delta\left(\boldsymbol{E}\left(\theta_{1}, \theta_{2}\right)\right)=\frac{\partial E_{1}}{\partial \theta_{1}}\left(\boldsymbol{F}\left(\theta_{1}, \theta_{2}\right)\right) \delta\left(\theta_{1}, \theta_{2}\right)+\epsilon\left(\boldsymbol{F}\left(\theta_{1}, \theta_{2}\right)\right) .
$$

The trivial fixed point $E_{1}\left(\theta_{1}, \theta_{2}\right)=F_{1}\left(\theta_{1}, \theta_{2}\right)=\theta_{1}$ has been analyzed by Stellan Ostlund [14]; it happens to occur at $a=b=\omega=0$ (point $R$ on fig. 15). The scale factor $A$ (eq. (3.3)) for this fixed point does not shear $(\beta=0)$ but the stretch in $\theta_{1}(\alpha)$ is arbitrary. To allow comparisons with the other fixed points, we tabulate the two values $\alpha= \pm \sigma^{-1}$. (The relevant eigenvalues one could generate using $|\alpha|<1$ are of no apparent interest.) The eigenvectors can be expanded as polynomials in $\theta_{1}$ and $\theta_{2}$. Ignoring the commutativity condition (3.9), for each $n, m$ there are two eigenvectors of (3.8) with degree $n$ in $\theta_{1}$ and $m$ in $\theta_{2}$; their eigenvalues are $\alpha^{1-n}(-\sigma)^{m+1}$ and $-\alpha^{1-n}(-\sigma)^{m-1}$. There are two relevant eigenvalues (unstable directions) and two marginal eigenvalues which satisfy (3.9) (see table I). The eigenvalue $\pm \sigma^{-2}$ governs the growth rate of any perturbation which does not leave the origin on an invariant curve. The eigenvalue $\sigma^{-1}$ governs perturbations which put the origin onto an invariant curve with $A \neq 0$; it expresses the increase of stability or instability of this curve under applications of $T$ (eq. (3.8)). The eigenvalue \pm 1 represents the marginally stable saddle-node line discussed below, where $(0,0)$ lies on an invariant curve with $A=0$. Finally, the eigenvalue $\mp 1$ tilts the invariant curve. As discussed above the coordinate transformation $B$ with $h \neq 0$ (skewing the coordinates) commutes with $A$ for $\alpha=-\sigma^{-1} ; h$ generates a one-parameter family of fixed points $E_{1}=\theta_{1}+h \sigma, F_{1}=\theta_{1}+h(\sigma-1)$. For $\alpha=\sigma^{-1}$ the tilt is reflected, leading to the eigenvalue 
Table I

Fixed points of $T$. For the trivial fixed point, the eigenvalues change sign ( \pm ) with $\alpha$. Sections of the table in brackets are conjectural

Fixed points of $T$ with $\theta_{1}$ rotation number $\rho_{1}=0$

Trivial fixed point $a=b=\omega=0$

$$
E_{1} \approx \theta_{1}, \quad F_{1}=\theta_{1}, \quad A=\left(\begin{array}{cc} 
\pm \sigma^{-1} & 0 \\
0 & -\sigma^{-1}
\end{array}\right)
$$

$\begin{array}{lll}\text { Eigenvalue } & \text { Eigenector } & \text { Significance } \\ \pm \sigma^{-2} & (c, \delta) & \\ \sigma^{-1} & (\sigma, 1) & \theta_{1} \text { rotation number } \\ \pm 1 & \left(\sigma \theta_{1}, 9_{1}\right) & \text { slope at fixed point } \\ \mp 1 & \left(\sigma \theta_{1}^{2}, \theta_{1}^{2}\right) & \text { curvature at fixed point }\end{array}$

$$
\begin{aligned}
& \text { Saddle-node fixed point } \\
& E_{1}=\theta_{1} /\left(1-\sigma c \theta_{1}\right), \quad F_{1}=\theta_{1} /\left(1-c \theta_{1}\right), \quad A=\left(\begin{array}{cc}
\sigma^{-1} & 0 \\
0 & -\sigma^{-1}
\end{array}\right) \\
& \text { Eigenvalue Significance } \\
& \sigma^{-2} \quad \text { saddle-node transition } \\
& 1 \text { change in } c \\
& -1 \quad \text { skew coordinates }
\end{aligned}
$$

Circle map fixed point

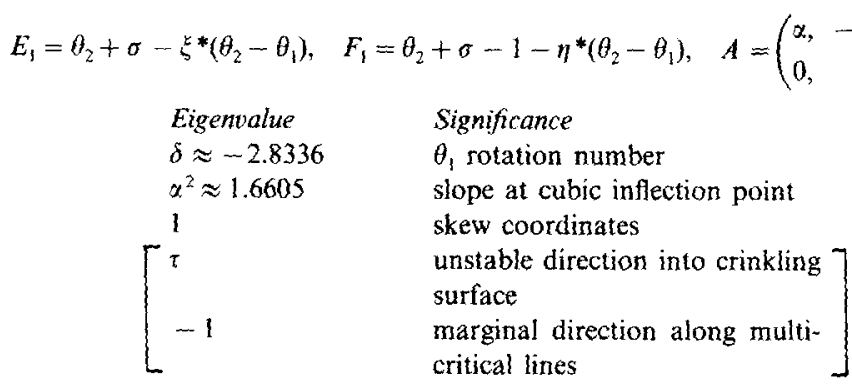

$\left[\begin{array}{ll} & \begin{array}{c}\text { Multicritical points (fixed points of } T^{2} \text { ) } \\ \text { One parameter family } E_{\mathrm{a}}, F_{\mathrm{a}}\end{array} \\ \text { Eigenvalue } & \multicolumn{1}{c}{\text { Significance }} \\ \delta_{a}^{2} & \text { fow into crinkling surface, other } \\ & \theta_{1} \text { rotation numbers } \\ \alpha_{a}^{4} & \text { flow down to saddle node, up to } \\ & \text { chaos } \\ 1 & \text { marginal direction, changes } a\end{array}\right]$

$\left.\left[\begin{array}{ll}\text { Eigenvalue } & \begin{array}{l}\text { Crinkling surface (fixed surface of } T \text { ) } \\ \kappa\end{array}\end{array}\right] \begin{array}{l}\text { Significance } \\ \text { fow away from surface (ergodic } \\ \text { average) }\end{array}\right]$


-1 , and a line of fixed points of $T^{2}$. No relevant eigenvalue whose eigenvector commutes (eq. (3.9)) depends upon $\theta_{2}$.

The stable manifold of the trivial fixed point intersects the three-parameter family of eq. (1.1) in the line connecting $\mathrm{C}$ to $\mathrm{R}$ in fig. 15. Perturbations in the $b$ direction appear to be irrelevant; thus only twc of the marginal and relevant eigendirections can be exhibited at once. On the one hand, if we expand about $\phi_{1}=0$ then changing $\omega$ keeping $a=0$ changes the rotation number (eigenvalue $\pm \sigma^{-2}$ ), while changing $\omega=-a$ moves along the saddle-node line $( \pm 1)$. On the other hand, if we expand about any $\phi_{1}$ value other than zero and $\pi$, the eigenvalues $\pm \sigma^{-2}$ and $\sigma^{-1}$ will be represented. The irrelevance of perturbations involving $\theta_{2}$ is interesting; near this fixed point iterations of the map gradually average out the $\theta_{2}$ dependence of any small additive perturbation.

The one-parameter family of saddle-node fixed points $\left\{E_{1}=\theta_{1} /\left(1-\sigma c \theta_{1}\right), F_{1}=\theta_{1} /\left(1-c \theta_{1}\right), \alpha=\sigma^{-1}\right.$, $\beta=0\}$ has been studied by several authors [6]. The eigenvalue $\sigma^{-2}$ gives the exponent $1 / 2$ quoted in section 2.2 which governs the intermittency and the contraction rate as one passes through the transition. The first marginal direction $(+1)$ noted in table I moves along the line of fixed points corresponding to the free constant $c$; the second marginal direction $(-1)$ tilts the invariant curve. (Again, technically we have a two-parameter family of saddle node fixed points, with various curvatures and tilts.) Although we have not performed the calculation, we believe that these are the only relevant and marginal eigenvalues; in particular we expect only irrelevant eigenvectors depend upon $\theta_{2}$. This implies again that $b$ gets averaged out under iterations and one sees the one-dimensional exponents as noted in section 2.2. The stable manifold of this family intersects fig. 15 in the surfaces $M_{1} C R S_{1}$ and $M_{2} C R S_{2}$. Here of course the lines $S_{i} R$ are not the fixed points, though they are independent of $\phi_{2}$.

As noted in section 2.3 , for $a=0$ eq. (1.1) is equivalent to the circle map in $\theta_{2}-\theta_{1}$, with the transition to chaos occurring at $b=1$. The associated fixed point $E_{1}=\theta_{2}+\sigma-\xi^{*}\left(\theta_{2}-\theta_{1}\right), F_{1}=\theta_{2}+\sigma-1-\eta^{*}$ $\left(\theta_{2}-\theta_{1}\right)$ (table I) is expressed in terms of the universal functions $\xi^{*}$ and $\eta^{*}$ of the circle map discussed in ref. 2. Both $\xi^{*}$ and $\eta^{*}$ have cubic inflection points at the origin $\left(\xi^{*}(\theta) \sim \eta^{*}(\theta) \sim \theta^{3}\right.$ as $\left.\theta \rightarrow 0\right)$. Two unstable directions exist which involve only $\theta_{2}-\theta_{1}$; the exponent $\delta$ governs perturbations which change the winding number $\rho_{1}$ in the $\theta_{1}$ direction, and the exponent $\alpha^{2}$ governs both the growth of the slope at the cubic inflection point and more generally the transition to chaos at fixed rotation number $\rho_{1}$. (Recall that our perturbations do not alter the $\theta_{2}$ evolution.) Again, there is a linear change of coordinates $B$ (with $g=-h$ ) which commutes with $A$, thus there will be a marginal directions corresponding to the family of fixed points found by skewing the coordinates by $B$.

We have now completed a summary of the known fixed points and important eigenvalues for our renormalization group. The remainder of this paper will become increasingly speculative; we will attempt to outline the type of results obtainable from a numerical implementation of the renormalization group 3.2.

The scaling law obeyed by the low frequency spectrum at the multicritical point (eq. (2.12) and figs. 9 and 10) indicates that self-similar behavior is occurring on all timescales, and that a RG fixed point should exist. However, fixed points of $T$ obey scaling laws [2] relating $\widetilde{f}(v)$ with $\widetilde{f}(\sigma v)$; the multicritical scaling law relates $\tilde{f}(v)$ with $\tilde{f}\left(\sigma^{2} v\right)$ (eq. (2.12)). The multicritical point will thus flow to fixed points of $T^{2}$ (two cycles of $T)$. We conjecture that a one-parameter family $\left(\boldsymbol{E}_{a}, \boldsymbol{F}_{a}\right)$ of multicritical fixed points of $T^{2}$ exists, whose low frequency spectra will be universal (with fractional corrections proportional to the frequency, as in ref. 2).

Since as $a \rightarrow 0,\left(\boldsymbol{E}_{a}, \boldsymbol{F}_{a}\right)$ approach the circle map fixed point, the latter will presumably have a marginal direction (whose eigenvalue of $T$ will be -1 ) leading along the multicritical lines. In analogy with the trivial 
fixed point there is probably at least one unstable direction from the circle map fixed point which is not a function of $\theta_{2}-\theta_{1}$; the corresponding exponent $\tau$ would govern perturbations (e.g., increasing $a$ ) leading onto the crinkling surface $\mathbf{M}_{1} \mathrm{CM}_{2}$.

Since we must vary two parameters in eq. (1.1) to find a multicritical point, it seems reasonable to presume that there are two relevant eigenvalues so that the stable manifold of the family of fixed points is of codimension two. Consider the unstable manifold of one of the multicritical points $M$. The contraction rate $\Lambda$ is zero at $\mathbf{M}$; it should be non-zero and negative in the mode-locked region of the unstable manifold. Along some curve passing through $\mathrm{M}$ the mode-locked state is undergoing a saddle-node bifurcation and $\Lambda=0$; by eq. (3.8) this curve is mapped onto itself and the tangent to it at $\mathrm{M}$ must be an eigenvector of $T^{2}$. It seems safe to assume that as $a \rightarrow 0$ the associated eigenvalue goes smoothly to the circle map exponent $\alpha^{4}$ (M is a fixedpoint of $T^{2}$ ), so we call this exponent $\alpha_{a}^{4}$. The multicritical fixed points will clearly also have two marginal directions. One, with eigenfunction $\partial\left(\boldsymbol{E}_{a}, \boldsymbol{F}_{a}\right) / \partial a$ will be associated with motion along the multicritical line $\mathrm{M}_{1} \mathrm{C}$ and $\mathrm{M}_{2} \mathrm{C}$ in fig. 13; the other will again correspond to that ratio of $g$ and $h$ which allows the coordinate transformation $B$ to commute with $A$ and thus with $T$.

There remains a second relevant eigenvalue at $M$ to be characterized. Numerical evidence (fig. 7) and the analysis of section 2.1 indicate that $A=0$ at the crinkling transition surface $\mathbf{M}_{1} \mathbf{C M}_{2}$ of fig. 15. Thus the crinkling boundary of the mode-locked region on the unstable manifold of $M$ should be mapped onto itself by $T^{2}$. If a tangent to the crinkling boundary exists at $\mathrm{M}$, it will be an eigenvector of $T^{2}$, presumably a relevant one. (Since we know only isolated points on the crinkling boundary, and those without great accuracy, we cannot be sure that it forms a smooth curve.) It is not clear from fig. 2 whether this eigenvector is independent of the saddle-node direction; the points at $b=1.145$ indicate that the crinkling curve and the intermittent tornado boundary may become tangent at M. For the analysis in the remainder of this section we shall assume that this eigenvector exists and is independent of the eigenvector corresponding to $\alpha_{a}^{4}$. We shall call the eigenvalue $\delta_{a}^{2}$, it is not obvious whether in the limit $a \rightarrow 0$ this eigenvalue will equal $\delta^{2}$ for the circle map.

The renormalization group transformation $T^{2}$ will, as noted above, map the crinkling surface in function space into itself. For the circle map [2], the unstable manifold corresponding to $\delta$ is mapped into itself by $T$, but the map is chaotic, depending in detail on the continued fraction expansion of the winding number [2]. Only if one controls the winding number as a relevant parameter can one find fixed points and exponents. It has been suggested in that system that most winding numbers (forming an ergodic set of Lebesgue measure one on which the map induced by $T$ acts ergodically) will possess "average" exponents [2]; there is no evidence for or against this suggestion. We believe the action of $T^{2}$ on the crinkling surface is also chaotic. The highly singular nature of the power spectrum at the crinkling transition and the lack of any observable scaling, with new structure on all timescales, indicates to us that at a minimum there are relevant parameters at the crinkling transition which we have not controlled. The operator $T^{2}$ carries points near the crinkling surface farther away, while it mixes points on the surface. We will assume for the remainder of this section that the unstable direction leading away from the crinkling surface characterized by an "average" eigenvalue $\kappa$ for all nearby points. (If in fig. 7 we crudely estimate $\Lambda(b) \sim\left(b-b_{\mathrm{c}}\right)$, then $\left.\kappa=\sigma^{-1}\right)$.

In fig. 8 we saw the crossover behavior of the exponent for the contraction rate $A$, from a value of about $1 / 3$ to the saddle-node value of $1 / 2$. If we assume $\delta_{a}^{2}>\alpha_{a}^{4}$, the multicritical value of the exponent for $A$ will depend on $\kappa$; the renormalization group flows will take one first along the crinkling surface (eigenvalue $\delta_{a}^{2}$ ) and then away from it (eigenvalue $\kappa$ ). [If $\delta_{a}^{2}<\alpha_{a}^{4}$, the same analysis will apply, exchanging $\left|\delta_{a}\right|$ for $\alpha_{a}^{2}$ and $\sigma^{-2}$ for $\kappa$.] Let $x$ be a measure of the distance in function space from the saddle-node surface, and 
$y$ be a distance from the crinkling surface. Then $\Lambda(x, y)$ satisfies (via eq. (3.8))

$$
\begin{aligned}
& y \sim 1, \quad \Lambda(\epsilon, y) \sim \epsilon^{1 / 2}, \\
& x \sim 1, \quad \Lambda(x, \epsilon) \sim \epsilon^{\log \sigma^{-1} / \log \mid \kappa !} .
\end{aligned}
$$

Near $\mathrm{M}(x=y=0)$ the $\mathrm{RG}$ flow is close to linear, and using eq. (3.8) we see

$$
\Lambda(x, y)=\sigma^{2} \Lambda\left(\delta_{a}^{2} x, \alpha_{a}^{4} y\right), \quad x, y \ll 1 .
$$

Approaching $\mathrm{M}$ in a transverse direction $\epsilon x_{0}, \epsilon y_{0}$, we find

$$
\begin{aligned}
\Lambda\left(\epsilon x_{0}, \epsilon y_{0}\right) & \approx \sigma^{-\log \epsilon / \log \left|\delta_{a}\right|} \Lambda\left(x_{0}, \alpha_{a}^{-2\left(\log \epsilon / \log \left|\delta_{a}\right|\right\}} \epsilon y_{0}\right) \\
& \approx \epsilon^{\log \sigma^{-1} / \log \left|\delta_{a}\right|} \epsilon^{\left(1-\log \alpha_{a}^{2} / \log \left|\delta_{a}\right|\right)\left(\log \sigma^{-1} / \log |\kappa|\right)} \\
& \approx \epsilon^{\left\{\log \sigma^{-1} / \log \left|\delta_{a}\right|+\log \sigma^{-1} / \log |\kappa|-\left(\log \alpha_{a}^{2} / \log \left|\delta_{a}\right|\right) \log \sigma^{-1} / \log |\kappa|\right\}} \approx \epsilon^{1 / 3} .
\end{aligned}
$$

Thus the exponent of the contraction rate $A$ along a transversal approach to the multicritical point (and thus the nontrivial exponent in fig. 8 before the crossover) can be written explicitly in terms of $\delta_{a}, \alpha_{a}$ and $\kappa$.

\section{Conclusion}

We would like to summarize the status of our understanding of this system. In this paper we have given a careful qualitative description of the phases and transitions observed in this system adjacent to a mode-locked state; other transitions can occur (e.g., "period doubling" of tori) for larger values of $b$ and $a$, but these seemed of less interest to us. We understand the saddle-node transition quantitatively and the boundedness of the chaotic "tornado" state as a partial breakdown of averaged equations of motion. We have demonstrated numerically the self-similar nature of the multicritical attractor and the scaling law for its spectrum, and have given an explicit criterion for locating it in terms of the Liapunov exponent $A$ and the zero Jacobian curve $F$ for the map. We have set up a renormalization group to study this multicritical point, and believe that a quantitative understanding of it will be obtained from a numerical implementation of this group.

There are two features of this system which are not under control. First, the crinkling transition appears to be a generic route to chaos in systems forced at two frequencies; it takes one continuously from a one-dimensional attractor to the pre- sumably two-dimensional tornado attractor. No quantitative understanding of this transition exists; indeed, we have not found an effective numerical algorithm to locate it. Secondly, the chaotic tornado attractor undergoes an intermittent breakdown; this transition is similar to "escape time" problems in other chaotic systems and might warrant further study.

\section{Acknowledgements}

The authors are indebted to D. Rand, D. Ruelle, and Stellan Ostlund for several helpful comments. Our research was supported by the National Science Foundation under Grant No. PHY77-27084 (supplemented by funds from the National Aeronautics and Space Administration) and the Department of Energy Grant No. DE AC02-83 ER 13044.

\section{References}

[1] M.J. Feigenbaum, J. Stat. Phys. 19 (1978) 25; 21 (1979) 669; Phys. Lett. 74A (1979) 375, Comm. Math. Phys. 77 (1980) 65.

[2] D. Rand, S. Ostlund, J. Sethna and E. Siggia, Phys. Rev. Lett. 49 (1982) 271; S. Ostlund, D. Rand, J. Sethna and E. Siggia, Physica 8D (1983) 303. 
[3] S. J. Shenker, Physica 5D (1982) 405. M.J. Feigenbaum, L.P. Kadanoff and S.J. Shenker, Physica 5D (1982) 370.

[4] D.F. Escande and F. Doveil, J. Stat. Phys. 26 (1981) 257. L.P. Kadanoff, Phys. Rev. Lett. 47 (1981) 1641; S.J. Shenker and L.P. Kadanoff, J. Stat. Phys. 27 (1982) 631.

[5] J.M. Greene, R.S. Mackay, F. Vivaldi and M.J. Feigenbaum, Physica 3D (1981) 486. P. Collet, J.-P. Eckmann and H. Koch, Physica 3D (1981) 457. M. Widom and L.P. Kadanoff, Physica 5D (1982) 287.

[6] J.E. Hirsch, M. Nauenberg and D.J. Scalapino, Phys. Lett. A87 (1982) 391. P. Manneville and Y. Pomeau, Phys. Lett. 75A (1979) 1; Commun. Math. Phys. 74 (1980) 189. A.B. Zisook, Phys. Rev. A 25 (1982) 2289; A.B. Zisook and S.J. Shenker, Phys. Rev. A25 (1982) 2824.

[7] D. Ruelle and F. Takens, Comm. Math. Phys. 20 (1971) 167; S.E. Newhouse, D. Ruelle and F. Takens, Comm. Math. Phys. 64 (1978) 35.

[8] J. Guckenheimer, "On Quasiperiodic Flow with Three Independent Frequencies", private communication.
[9] V.I. Arnold, Translations American Mathematical Society, Second Series 46 (1965) 213. M.R. Herman, "Sur la Conjugasons Differentiable des Diffeomorphismes du Cercle à des rotations", Publ. I.H.E.S. 49 (1979) 5; in: Geometry and Topology, Springer Lecture Notes in Mathematics (J. Palis and M. do Carmo, eds.) 597 (1977) 271.

[10] D. Rand, Arch. Rat. Mech. Anal. 79 (1982) 1. M. Gorman, H.L. Swinney and D. Rand, Phys. Rev. Lett. 46 (1981) 992.

[11] H. Rüssman, Ann. of the NYAS 357 (1980) 90. E.I. Dinaburg and Ya.G. Sinai, Functional Anal. Appl. 9 (1975) 279. R. Johnson and J. Moser, Comm. Math. Phys. 84 (1982) 403.

[12] J.M. Greene, J. Math. Phys. 20 (1979) 1183.

[13] J.L. Kaplan and J.A. Yorke, Functional Differential Equations and Approximations of Fixed Points, H.O. Peitgen and H.O. Walther, eds. (Springer, Notes in Math. \# 730 (1979) 228.

[14] Stellan Ostlund, private communication. 